\title{
Expression of circ_001569 is upregulated in osteosarcoma and promotes cell proliferation and cisplatin resistance by activating the $W n t / \beta$-catenin signaling pathway
}

\author{
HONGFEI ZHANG, JIAPENG YAN, XUJIAN LANG and YUESHENG ZHUANG \\ Department of Orthopedics, Affiliated Hospital of Weifang Medical University, \\ Kuiwen, Weifang, Shandong 261031, P.R. China
}

Received November 2, 2017; Accepted April 12, 2018

DOI: $10.3892 / \mathrm{ol} .2018 .9410$

\begin{abstract}
Circular RNAs (circRNAs), a type of non-coding RNAs derived from back-splicing, havebeen reported to function as gene expression regulators involved in tumor development of multiple human tumors. However, the clinical significance and underlying molecular mechanisms of circ_001569 in osteosarcoma still be unknown. In the study, we found that circ_001569 expression was significantly overexpressed in osteosarcoma tissues compared with adjacent noncancerous bone tissues. Higher circ_001569 expression significantly correlated with distant metastasis and advanced tumor stage of osteosarcoma patients. Gain-function and loss-function assays showed that circ_001569 knockdown significantly inhibited osteosarcoma cell proliferation and cell colon formation capacities. Moreover, upregulation of circ_001569 significantly promoted osteosarcoma cell resistance to cisplatin by activating $\mathrm{Wnt} / \beta$-catenin signaling pathway. Thus, these results indicated that circ_001569 represented a novel potentially therapeutic target of osteosarcoma.
\end{abstract}

\section{Introduction}

Osteosarcoma (OS) is one of the most common primary bone tumors and accounts for approximately 5-6\% in all tumors of childhood and adolescence $(1,2)$. Recently, larger advanced in therapeutic methods for the disease including tumor resection, neoadjuvant or adjuvant chemotherapy and radiotherapy, patients have prolonged the overall survival time (3). However, due to the aggressive potential of osteosarcoma, $40 \%$ develop to metastasis at advanced stages during treatment for patients (4). Thus, revealing the molecular mechanisms

Correspondence to: Dr Jiapeng Yan, Department of Orthopedics, Affiliated Hospital of Weifang Medical University, 2428 Yuhe Road, Kuiwen, Weifang, Shandong 261031, P.R. China

E-mail: zhfwfyxy@163.com

Key words: osteosarcoma, circular RNA, circ_001569, $\mathrm{Wnt} / \beta$-catenin, chemotherapy resistance underlying osteosarcoma development is important for osteosarcoma treatment.

Circular RNAs are newly discovered endogenous non-coding RNAs characterized by their covalently closed loop structures without a 5'cap or a 3'Poly A tail (5). Studies have suggested that circular RNAs play important roles in various diseases including cancer. CircRNAs act as oncogenes or tumor suppressors to be involved in carcinogenesis by regulating gene expression at the transcriptional or post-transcriptional level $(6,7)$. For instance, Chen et al reported that CircRNA_100290 is upregulated and co-expressed with CDK6 in oral cancer and functions as a competing endogenous RNA to regulate CDK6 expression through sponging miR-29b family members (8). Hsa_circ_001569 is higher expression in colorectal cancer and identified as a sponge of miR-145 targeting E2F5, BAG4 and FMNL2 to promote cell proliferation and invasion of colorectal cancer (9). However, the clinical significance and function of circ_001569 in osteosarcoma remains larger unknown.

In the present study, we found that circ_001569 was significantly overexpressed in osteosarcoma and correlated with distant metastasis, advanced tumor stage, and poor prognosis of osteosarcoma. Knockdown of circ_001569 significantly inhibited the proliferation ability of osteosarcoma. Moreover, upregulation of circ_001569 significantly promoted osteosarcoma cell resistance to cisplatin by activating Wnt/ $\beta$-catenin signaling pathway. Thus, our results indicated that circ_001569 may serve as a novel potentially therapeutic target of osteosarcoma.

\section{Patients and methods}

Patient tissues samples. A total of 36 primary osteosarcoma tissue samples and adjacent non-cancerous bone tissue samples were obtained from the Department of Orthopedics, Affiliated Hospital of Weifang Medical University (Weifang, China) between January 2009 and February 2013. The patients included 23 males and 13 females, with a median age of 35.8 years and an age range of 10 to 56 years old. The histological grade was assessed according to the tumor-node-metastasis (TNM) system (10). None of the patients received any treatment prior to surgery. The removed 
fresh tissues were immediately placed in liquid nitrogen and then stored at $-80^{\circ} \mathrm{C}$ for RNA analysis. The clinicopathological data are summarized in Table I. Written informed consent was obtained from all patients and the study was approved by the Ethics Committee of Affiliated Hospital of Weifang Medical University.

Cell line culture. Two human osteosarcoma cell lines (MG-63 and U2OS) and a normal human osteoblastic cell line hFOB were obtained from Type Culture Collection of the Chinese Academy of Sciences (Shanghai, China). The hFOB cells were cultured in Dulbecco's modified Eagle's medium (DMEM) supplemented with $10 \%$ fetal bovine serum (FBS; Gibco; Thermo Fisher Scientific, Inc., Waltham, MA, USA). The human OS cells were cultured in RPMI-1640 medium supplemented with 10\% FBS (both Gibco; Thermo Fisher Scientific, Inc.), $100 \mathrm{U} / \mathrm{ml}$ penicillin and $100 \mathrm{mg} / \mathrm{ml} \mathrm{streptomycin}$ (Thermo Fisher Scientific, Inc.). Cells were incubated at $37^{\circ} \mathrm{C}$ with $5 \% \mathrm{CO}_{2}$ in a humidified chamber.

Cell transfection and plasmid construct. siRNA targeting has-circ_001569 (5'-GCATCGTGCAGGACTGGAA-3') and si-negative control (NC; 5'-UUCUCCGAACGUGUCACG UTT-3') was constructed and purchased from Guangzhou RiboBio Co., Ltd. (Guangzhou, China). Cells were transiently transfected with $100 \mathrm{nmol} / \mathrm{l}$ oligoribonucleotides using Lipofectamine 2000 (Thermo Fisher Scientific, Inc.), according to the manufacturer's protocol. Human hsa_circ_001569 cDNA was synthesized and cloned into pLVX-IRESneo by GeneCopoeia, Inc. (Rockville, MD, USA). Cells were transfected with the oligonucleotides at room temperature at a final concentration of $20 \mathrm{nmol} / \mathrm{l}$ using Lipofectamine 2000 (Thermo Fisher Scientific, Inc.) according to the manufacturer's protocol. Cells were harvested following cell transfection at $48 \mathrm{~h}$.

Reverse transcription-quantitative polymerase chain reaction (RT-qPCR). Total RNA was extracted from tissues and cells using TRIzol reagent (Thermo Fisher Scientific, Inc.). Reverse Transcription kit (Guangzhou RiboBio Co., Ltd.) was used to reverse RNA to cDNA, according to the manufacturer's protocols. RT-qPCR was performed using a SYBR Premix Ex TaqTM II kit (Takara Biotechnology Co., Ltd., Dalian, China) on an ABI7500 system (Applied Biosystems; Thermo Fisher Scientific, Inc.). The thermocycling conditions were as follows: $95^{\circ} \mathrm{C}$ for $10 \mathrm{~min}$, followed by 40 cycles of $95^{\circ} \mathrm{C}$ for $10 \mathrm{sec}, 57^{\circ} \mathrm{C}$ for $20 \mathrm{sec}$ and $72^{\circ} \mathrm{C}$ for $15 \mathrm{sec}$. The mRNA fold-change of circ_001569 was calculated by the $2^{-\Delta \Delta \mathrm{Cq}}$ method (11) and was normalized to GAPDH mRNA expression. The primer sequences were as follows: circ_001569 forward, 5'-TCCCCTGAACATTCTCCC CAT-3' and reverse, 5'-GAAAGCACTTGGTGAAGTCGG-3'; and GAPDH forward, 5'-CACCGTAGCCTTCCGAGTA-3' and reverse, 5'-GCCCTTGATGAGCTGTTGA-3'.

Cell proliferation assay. Cell proliferation capacity was evaluated using the Cell Counting kit-8 (CCK-8; Dojindo Molecular Technologies, Inc., Kumamoto, Japan) according to the manufacturer's protocol. Transfected cells were seeded onto 96-well plates $\left(2,000\right.$ cells/well) and were incubated overnight at $37^{\circ} \mathrm{C}$ with $5 \% \mathrm{CO}_{2}$ in a humidified chamber. The proliferation rate of osteosarcoma cells was detected every 24 h until 72 h. Each well was supplemented with $10 \mu \mathrm{l}$ CCK- 8 regent and cells were incubated for $2 \mathrm{~h}$. Next, cell proliferation was measured at $450 \mathrm{~nm}$ using a Multiskan MK3 spectrophotometer.

Cell colony formation assay. The transfected osteosarcoma cells were plated at 500 cells/well onto 6-well plates. After cells were cultured at $37^{\circ} \mathrm{C}$ with $5 \% \mathrm{CO}_{2}$ in a humidified chamber for two weeks, the cell colonies were fixed with $4 \%$ paraformaldehyde at room temperature for $20 \mathrm{~min}$ and stained with $0.1 \%$ crystal violet at room temperature for $20 \mathrm{~min}$, and the images of cell colonies were captured and cell colonies were calculated.

Western blot analysis. Total protein was extracted using lysis buffer (Sigma-Aldrich; Merck KGaA, Darmstadt, Germany). A BCA kit (Tiangen Biotech Co., Ltd., Beijing, China) was used to detect the concentration of the proteins. Equal protein $(30 \mu \mathrm{g})$ was separated on $8-12 \%$ SDS-PAGE gel and transferred onto polyvinylidene difluoride membranes (Millipore, Billerica, MA, USA). Following blocking with 5\% skimmed milk at room temperature for $1 \mathrm{~h}$, the membrane was incubated with antibodies including p-GSK-3 $\beta$ (cat. no. 9336; 1:1,000), GSK-3 $\beta$ (cat. no. 9315; 1:2,000), $\beta$-catenin (cat. no. 9562; 1:1,000) and GAPDH (cat. no. 5174; 1:1,000; all Cell Signaling Technology, Inc., Danvers, MA, USA) overnight at $4^{\circ} \mathrm{C}$ and then incubated with horseradish peroxidase-conjugated goat anti-rabbit antibody (cat. no. 10285-1-AP; 1:1,000; ProteinTech Groups, Inc., Chicago, IL, USA) at room temperature for $1 \mathrm{~h}$. The membrane was detected with an enhanced chemiluminescence detection system (Nanjing KeyGen Biotech Co., Ltd.). GAPDH was used as an internal control.

The value of $50 \%$ cytotoxic concentration (IC50) in osteosarcoma cells. The cells were transfected with si-NC and si-circ_001569 or pLVX-IRESneo-vector (vector) and pLVX-IRESneo-circ_001569 (circ_001569). Cell viability was detected using CCK-8, according to the manufacturer's protocol. The IC50 values were defined as the chemotherapeutic agent concentration producing $50 \%$ inhibition of cell proliferation. Absorbance was detected at $450 \mathrm{~nm}$.

Statistical analysis. All data in the study are presented as the mean \pm standard deviation and were analyzed using SPSS 19.0 software package (IBM Corp., Armonk, NY, USA). Student's t-test was applied to compare the differences between two groups. For comparisons among 3 or more groups, one-way analysis of variance, followed by the Student-Newman-Keuls post hoc test, was used. $\mathrm{P}<0.05$ was considered to indicate a statistically significant difference.

\section{Results}

Expression of circ_001569 is elevated in osteosarcoma. To investigate the clinical significance of circ_001569 expression in osteosarcoma, the present study examined the expression of circ_001569 by RT-qPCR in 36 primary osteosarcoma tissues and adjacent non-cancerous bone tissues. As demonstrated in Fig. 1A, circ_001569 expression was significantly elevated in osteosarcoma tissues compared with adjacent non-cancerous bone tissues $(\mathrm{P}<0.05)$. Next, the association between 
Table I. Association between circ_001569 expression and clinical characteristics in 36 patients with osteosarcoma.

circ_001569 expression

Clinical characteristic

Total $(n=36)$

Lower $(n=16)$

Higher $(n=20)$

P-value

Sex
Female

13

23

Male

Age, years

$\leq 18$

26

$>18$

10

Site

Femur

18

Tibia

10

Humerus

8

Distant metastasis

$\begin{array}{ll}\text { Absent } & 16 \\ \text { Present } & 20\end{array}$

Present

Tumor size, $\mathrm{cm}$

$\begin{array}{ll}<5 & 14 \\ \geq 5 & 22\end{array}$

$\geq 5$

22

TNM stage

I-IIA

14

IIB-III

\section{8}

8

11

5

0.799

7

5

4

11

5

$8 \quad 6$

$8 \quad 14$

$10 \quad 4$

$6 \quad 16$
0.121

${ }^{\mathrm{a}} \mathrm{P}<0.05$.
A

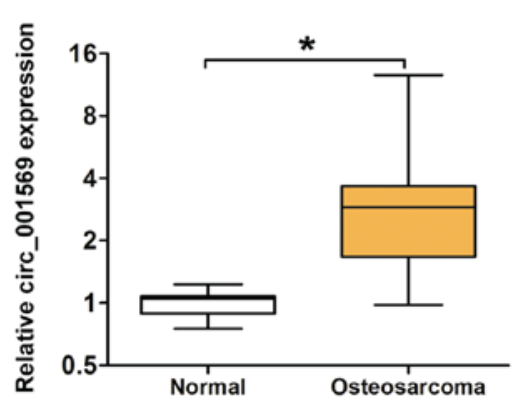

C

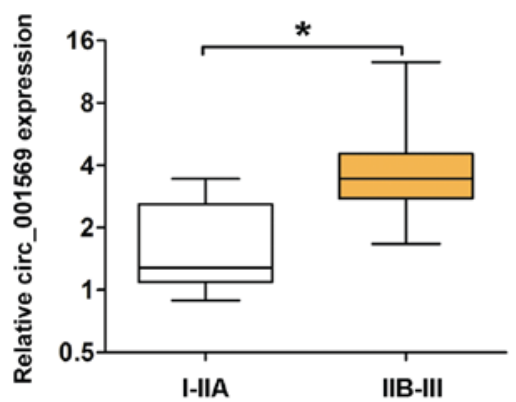

B

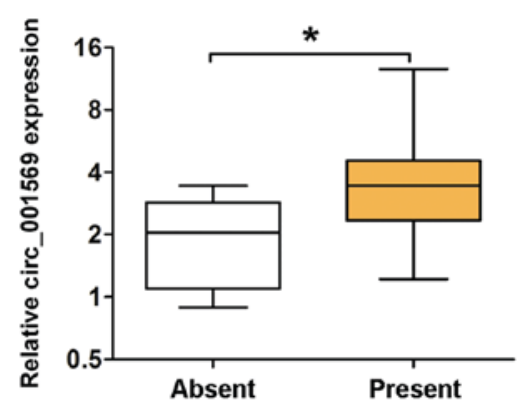

D

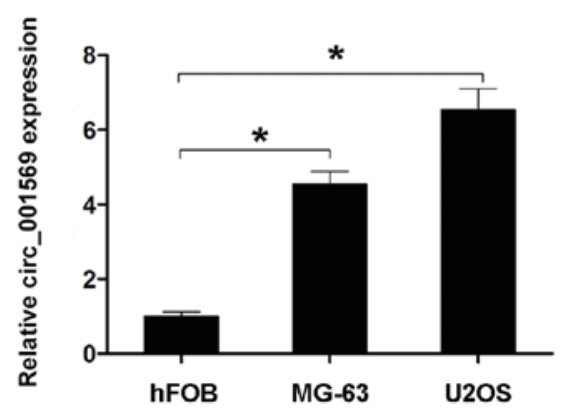

Figure 1. Expression of circ_001569 is higher in osteosarcoma. (A) The relative expression of circ_001569 was detected using RT-qPCR in 36 primary osteosarcoma tissue samples and adjacent non-cancerous bone tissue samples. (B) Association between circ_001569 expression and distant metastasis in osteosarcoma patients. (C) Association between circ_001569 expression and Tumor-Node-Metastasis stage in patients with osteosarcoma. (D) The relative expression of circ_001569 was detected using RT-qPCR in two human osteosarcoma cell lines (MG-63 and U2OS) and a normal human osteoblastic cell line, hFOB. Data are presented as the mean \pm standard deviation. ${ }^{*} \mathrm{P}<0.05$. RT-qPCR, reverse transcription-quantitative polymerase chain reaction. 
A

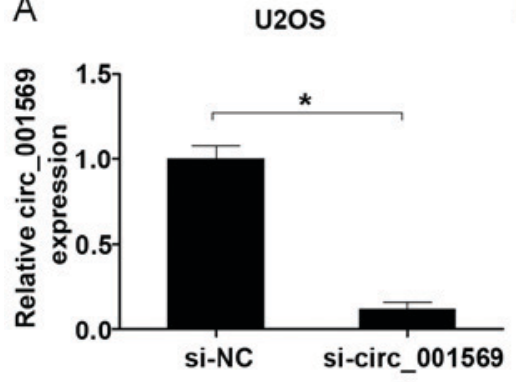

D

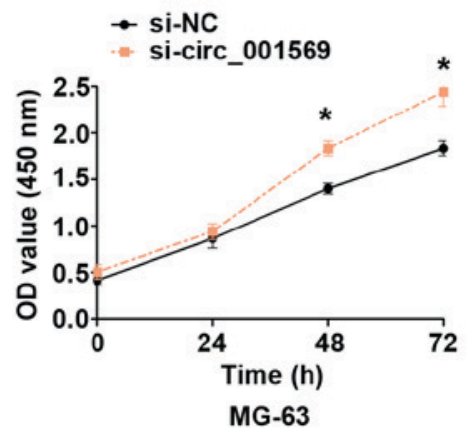

B

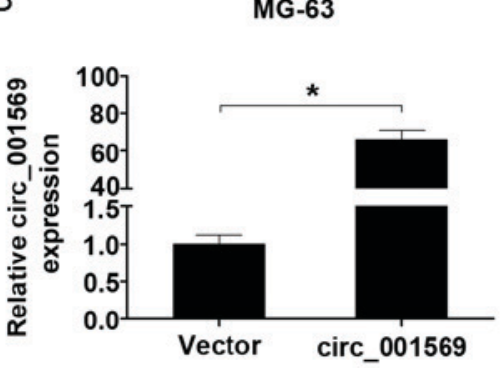

E
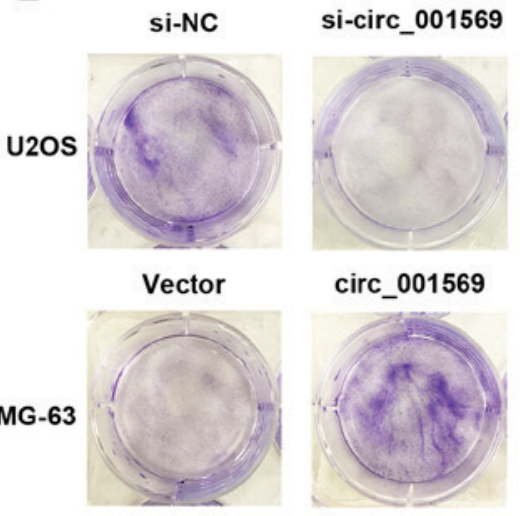

c

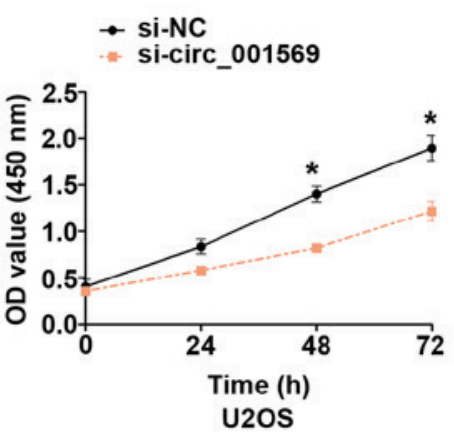

F

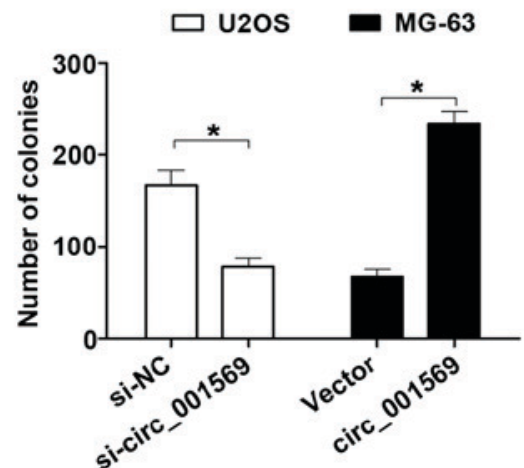

Figure 2. Upregulation of circ_001569 expression enhances cell proliferation ability in vitro. (A) The relative expression of circ_001569 was detected using RT-qPCR following transfection with si-NC or si-circ_001569 in U2OS cells. (B) The relative expression of circ_001569 was detected using RT-qPCR following transfection with empty vector or overexpressed circ_001569 plasmid in MG-63 cells. (C) Cell proliferation ability was detected using CCK8 assay following transfection with si-NC or si-circ_001569 in U2OS cells. (D) Cell proliferation ability was detected using CCK8 assay following transfection with empty vector or overexpressed circ_001569 plasmid in MG-63cells. (E) Cell proliferation was detected using cell colony formation assay following transfection with si-NC or si-circ_001569 in U2OS cells. (F) Cell proliferation was detected using a cell colony formation assay following transfection with empty vector or overexpressed circ_001569 plasmid in MG-63 cells. Data are presented as the mean \pm standard deviation. "P $<0.05$. RT-qPCR, reverse transcription-quantitative polymerase chain reaction; si, small interfering RNA; NC, negative control; CCK8, Cell Counting kit-8.

circ_001569 expression and clinicopathological factors was analyzed. The results of the present study demonstrated that circ_001569 expression was associated with distant metastasis (Fig. 1B; Table I, P<0.05) and TNM stage (Fig. 1C; Table I, $\mathrm{P}<0.05$ ), but was not associated with any others factors in osteosarcoma patients (Table I, $\mathrm{P}>0.05$ ). In addition, the expression of circ_001569 was detected in two human osteosarcoma cell lines (U2OS and MG-63) and an osteoblast cell line (hFOB) by RT-qPCR analyses. The results revealed that expression of circ_001569 was higher in osteosarcoma cell lines than that in hFOB cell lines (Fig. 1D). These results indicated that circ_001569 expression was upregulated in osteosarcoma.

Upregulation of circ_001569 expression enhances cell proliferation ability in vitro. To evaluate the effects of circ_001569 on cell proliferation, a loss-function assay was performed in U2OS cells and a gain-function assay was performed in MG-63 cells according to their expression of circ_001569 (Fig. 2A and B). CCK-8 assay results revealed that the cell proliferation capacity was significantly inhibited following circ_001569 knockdown compared with the negative control group in U2OS cells, but was notably enhanced following circ_001569 overexpression in MG-63 cells, compared with the control group (Fig. 2C and D). Cell colony formation assay revealed that the number of colonies formed in the circ_001569 knockdown group was smaller than that in the negative control group in U2OS cells, but was more when circ_001569 was overexpressed in MG-63 cells (Fig. 2E and F). Therefore, these results indicated that upregulation of circ_001569 could enhance cell proliferation in vitro.

Upregulation of circ_001569 promotes osteosarcoma cell resistance towards chemotherapy and activates the Wnt/ $\beta$-catenin signaling pathway. Combination of cisplatin with doxorubicin and methotrexate is frequently used for osteosarcoma treatment $(12,13)$. Furthermore, the present study evaluated whether circ_001569 expression affected conventional chemotherapy of osteosarcoma. The results indicated that inhibition of circ_001569 activity in U2OS cells resulted in a significant decrease in the calculated IC50 value for cisplatin, doxorubicin or methotrexate, compared with the control group (Fig. 3A). However, overexpression of circ_001569 activity in MG-63 cells enhanced the IC 50 value for cisplatin, doxorubicin or methotrexate (Fig. 3B). These results indicated that upregulation of circ_001569 promoted osteosarcoma cell resistance towards chemotherapy.

Upregulation of circ_001569 promotes osteosarcoma cell cisplatin resistance by activating the Wnt/ $\beta$-catenin signaling pathway. Activation of $\mathrm{Wnt} / \beta$-catenin signaling is associated with chemotherapy resistance in osteosarcoma (14). 
A

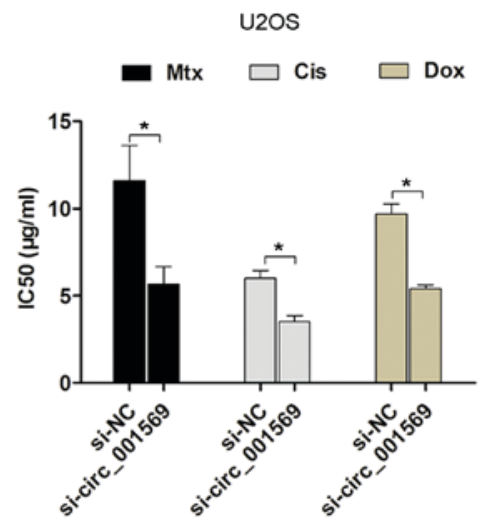

C

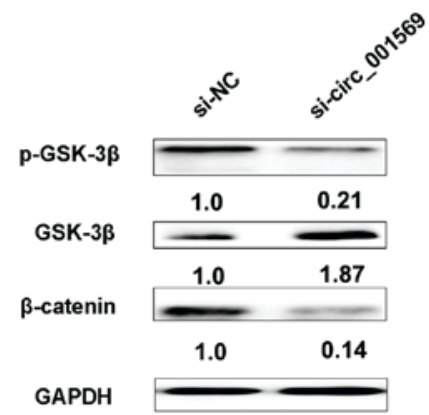

U2OS
B

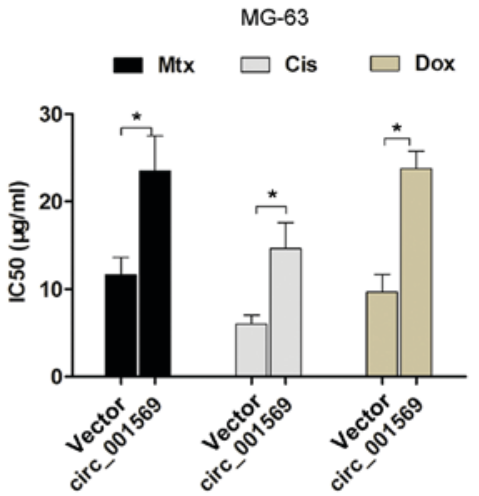

D

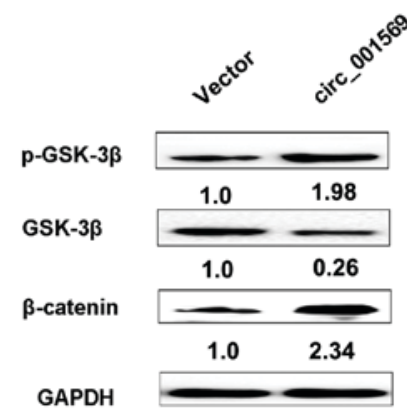

MG-63

Figure 3. Upregulation of circ_001569 promotes osteosarcoma cell resistance towards chemotherapy and activates Wnt/ $\beta$-catenin signaling. (A) Inhibition of circ_001569 activity in U2OS cells resulted in a significant decrease of the IC50 value for cisplatin, doxorubicin or methotrexate, compared with the control group. (B) Overexpression of circ_001569 activity in MG-63 cells could enhance the IC50 value for cisplatin, doxorubicin or methotrexate. (C) The relative expression of p-GSK-3 $\beta$, GSK-3 $\beta$ and $\beta$-catenin was detected using western blot analysis following transfection with si-NC or si-circ_001569 in U2OS cells. (D) The relative expression of p-GSK-3 $\beta$, GSK-3 $\beta$ and $\beta$-catenin was detected using western blot analysis following transfection with empty vector or overexpressed circ_001569 plasmid in MG-63 cells. Data are presented as the mean \pm standard deviation. ${ }^{*} \mathrm{P}<0.05$. IC50, calculated 50\% inhibition of growth; si, small interfering RNA; NC, negative control.

Furthermore, western blot analysis revealed that circ_001569 knockdown in U2OS cells decreased the expression levels of p-GSK-3 $\beta$ and $\beta$-catenin, but increased GSK-3 $\beta$ expression levels (Fig. 3C). However, circ_001569 overexpression increased the expression levels of p-GSK-3 $\beta$ and $\beta$-catenin in MG-63 cells, but decreased GSK-3 $\beta$ expression levels (Fig. 3D). These results indicated that circ_001569 overexpression activated the $\mathrm{Wnt} / \beta$-catenin signaling pathway.

Next, the present study demonstrated whether circ_001569 overexpression enhanced the resistance of osteosarcoma to cisplatin, doxorubicin or methotrexate by activating the Wnt $/ \beta$-catenin signaling pathway. As demonstrated in Fig. $4 \mathrm{~A}$ and $\mathrm{B}$, the Wnt $/ \beta$-catenin inhibitor, XAV939, or Wnt/ $\beta$-catenin agonist, $\mathrm{LiCl}$, could reduce or increase the IC50 value for cisplatin, doxorubicin or methotrexate. It was further demonstrated that circ_001569 knockdown resulted in a decreased IC50 value for cisplatin, doxorubicin or methotrexate, compared with the negative control. However, this chemotherapy sensitivity was only rescued by treatment with the Wnt/ $\beta$-catenin agonist, $\mathrm{LiCl}$ in the cisplatin-treated group, compared with the control group (Fig. 4C). Additionally, it was demonstrated that circ_001569 overexpression exhibited an increased IC50 value for cisplatin, doxorubicin or methotrexate, compared with the negative control. However, this chemotherapy resistance was also rescued by treatment with the Wnt/ $/$-catenin agonist, $\mathrm{LiCl}$, in the cisplatin-treated group, compared with the control group (Fig. 4D). Therefore, our aforementioned results indicated that circ_001569 could enhance cisplatin resistance of OS cells via activating the Wnt/ $\beta$-catenin pathway.

\section{Discussion}

Recent studies have reported that circRNAs serve as important regulators in cancer biological functions, including cell proliferation, migration, invasion and metastasis $(15,16)$. In a previous study, circ-0016347 was identified as a sponge of miR-214 that upregulates the expression of caspase- 1 to promote proliferation and invasion (17). The cir-GLI2 exerts tumor-promoting effects on the proliferation, migration and invasion of osteosarcoma cells via negatively targeting miR-125b-5p (18). The expression of hsa_circ_0,01564 is upregulated in osteosarcoma and hsa_circ_001564 knockdown significantly suppresses proliferation activity, induces cell-cycle arrest in the G0/G1 phase, and promotes the apoptosis of HOS and MG-63 cells (19). The present study demonstrated that circ_001569 was significantly overexpressed in osteosarcoma tissues compared with adjacent non-cancerous bone tissues. Higher circ_001569 expression was significantly associated with distant metastasis and advanced tumor stage in OS patients. Furthermore, circ_001569 overexpression enhanced cell proliferation and cell colony formation capacities; however, circ_001569 knockdown inhibited cell proliferation and cell colony formation abilities. These findings indicated 
A

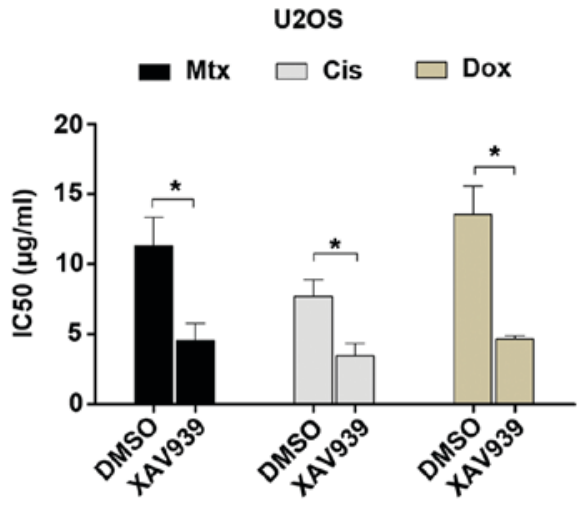

C

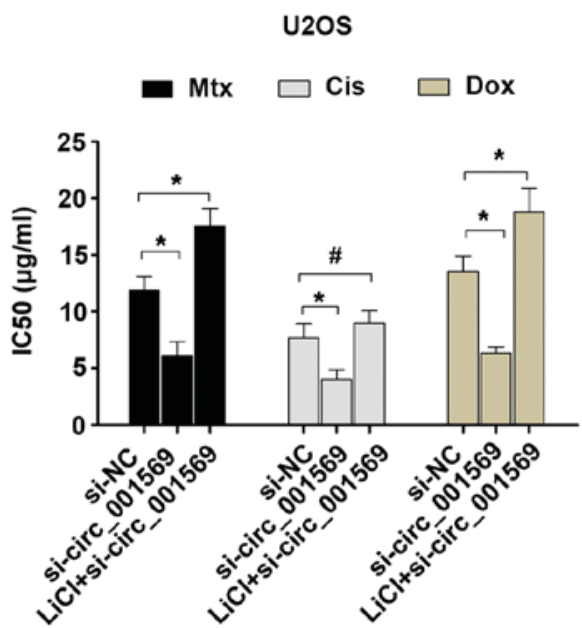

B

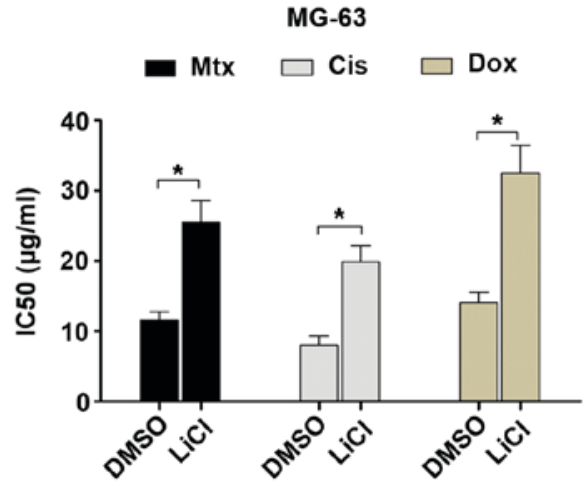

D

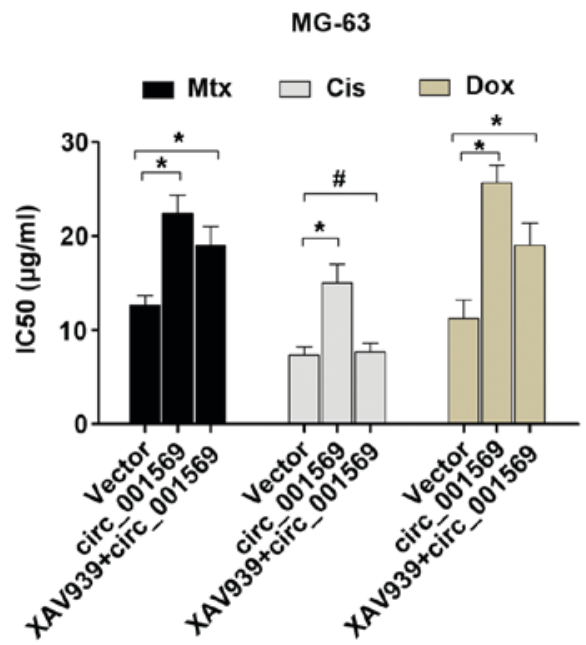

Figure 4. Upregulation of circ_001569 promotes resistance of osteosarcoma cells to cisplatin by activating the Wnt/ $\beta$-catenin signaling pathway. (A) The Wnt/ $\beta$-catenin inhibitor, XAV939, or (B) Wnt/ $\beta$-catenin agonist, LiCl, could reduce or increase the IC50 value for cisplatin, doxorubicin or methotrexate. (C) The IC50 value for cisplatin, doxorubicin or methotrexate in U2OS cells was detected following treatment of the cells with si-NC, si-circ_001569 or Wnt/ $\beta$-catenin agonist $(\mathrm{LiCl})+$ si-circ_001569. (D) The IC50 value for cisplatin, doxorubicin or methotrexate in MG-63 cells was detected following the treatment of cells with vector, circ_001569, or Wnt/ $\beta$-catenin inhibitor (XAV939) + circ_001569. Data are presented as the mean \pm standard deviation. ${ }^{*} \mathrm{P}<0.05$, \#, not statistically significant. IC50, calculated $50 \%$ inhibition of growth; si, small interfering RNA; NC, negative control.

that circ_001569 serves a promoting role in osteosarcoma development.

Previous studies have revealed that activation of Wnt/ $\beta$-catenin signaling could enhance chemotherapy resistance to cisplatin, doxorubicin and methotrexate. For example, TWIST decreases osteosarcoma cell survival against cisplatin by decreasing the soluble $\beta$-catenin level through a PI3K-dependent manner (14). Hypoxic osteosarcoma cells can be sensitized to doxorubicin treatment by further inhibition of the Wnt/ $\beta$-catenin signaling pathway (20). Overexpression of long non-coding RNA HOTTIP increases chemoresistance of osteosarcoma cells against cisplatin, doxorubicin and methotrexate by activating the $\mathrm{Wnt} / \beta$-catenin pathway (21). The present study demonstrated that circ_001569 knockdown decreased the expression levels of $\mathrm{p}-\mathrm{GSK}-3 \beta$ and $\beta$-catenin, but upregulated GSK-3 $\beta$ expression levels, while circ_001569 overexpression had an opposite effect. Furthermore, the present study demonstrated that osteosarcoma cells treated with Wnt/ $\beta$-catenin inhibitor, XAV939, reduced resistance to cisplatin, doxorubicin and methotrexate. However, osteosarcoma cells treated with the Wnt/ $\beta$-catenin agonist, $\mathrm{LiCl}$, promoted cell resistance to cisplatin, doxorubicin and methotrexate. Furthermore, it was demonstrated that the cell resistance to cisplatin caused by circ_001569 overexpression could be partially reversed by the combination treatment of circ_001569 overexpression and the Wnt/ $\beta$-catenin inhibitor, XAV939. In line with these results, the enhanced chemotherapy sensitivity induced by circ_001569 knockdown could be partially reversed by the combination treatment of circ_001569 knockdown and the Wnt/ $\beta$-catenin agonist, $\mathrm{LiCl}$. Therefore, these results indicated that circ_001569 promoted cell resistance to cisplatin in osteosarcoma. However, the mechanism by which circ_001569 regulates Wnt/ $\beta$-catenin signaling remains unknown. The classic role for circRNAs is to serve as an miRNA sponge or to interact with molecular proteins including GFRA1 and Foxo3, and act as ceRNAs in triple negative breast cancer by regulating miR-34a and Foxo3 activity promoted by non-coding effects of circular RNA and Foxo3 pseudogene in the inhibition of tumor growth and angiogenesis $(22,23)$. Therefore, revealing the underling mechanisms of circ_001569 involved in Wnt/ $\beta$-catenin signaling will be investigated in the future.

In conclusion, the results of the present study demonstrated that circ_001569 expression was higher in osteosarcoma. Upregulation of circ_001569 promoted cell proliferation ability. Furthermore, it was demonstrated that circ_001569 promoted 
cell resistance to cisplatin in osteosarcoma by activating Wnt $/ \beta$-catenin signaling. These results indicated that circ_001569 may be a potential therapeutic target of osteosarcoma.

\section{Acknowledgements}

Not applicable.

\section{Funding}

No funding was received.

\section{Availability of data and materials}

The datasets used and/or analyzed during the current study are available from the corresponding author on reasonable request.

\section{Authors' contributions}

HZ and JY conceived and designed the study. JY, XL and YZ performed the experiments. JY, XL and YZ analyzed and interpreted the data. YZ wrote the manuscript.

\section{Ethics aproval and consent to participate}

Written informed consent was obtained from all patients and the study was approved by the Ethics Committee of Affiliated Hospital of Weifang Medical University.

\section{Patient consent for publication}

All patients provided consent for the publication of their data.

\section{Conflicts of interests}

The authors declare that they have no competing interests.

\section{References}

1. Eppert K, Wunder JS, Aneliunas V, Kandel R and Andrulis IL: Von Willebrand factor expression in osteosarcoma metastasis. Mod Pathol 18: 388-397, 2005.

2. Mirabello L, Troisi RJ and Savage SA: Osteosarcoma incidence and survival rates from 1973 to 2004: Data from the surveillance, epidemiology and end results program. Cancer 115: 1531-1543, 2009.

3. Geller DS and Gorlick R: Osteosarcoma: A review of diagnosis, management, and treatment strategies. Clin Adv Hematol Oncol 8: 705-718, 2010.

4. Thompson LD: Osteosarcoma. Ear Nose Throat J 92: 288: 290-290, 2013.
5. Meng S, Zhou H, Feng Z, Xu Z, Tang Y, Li P and Wu M: CircRNA: Functions and properties of a novel potential biomarker for cancer. Meng S 16: 94, 2017.

6. Hansen TB, Jensen TI, Clausen BH, Bramsen JB, Finsen B, Damgaard CK and Kjems J: Natural RNA circles function as efficient microRNA sponges. Nature 495: 384-388, 2013.

7. Yang Z, Xie L, Han L, Qu X, Yang Y, Zhang Y, He Z, Wang Y and Li J: Circular RNAs: Regulators of cancer-related signaling pathways and potential diagnostic biomarkers for human cancers. Theranostics 7: 3106-3117, 2017.

8. Chen L, Zhang S, Wu J, Cui J, Zhong L, Zeng L and Ge S: circRNA_100290 plays a role in oral cancer by functioning as a sponge of the miR-29 family. Oncogene 36: 4551-4561, 2017.

9. Xie H, Ren X, Xin S, Lan X, Lu G, Lin Y, Yang S, Zeng Z, Liao W, Ding YQ and Liang L: Emerging roles of circRNA_001569 targeting miR-145 in the proliferation and invasion of colorectal cancer. Oncotarget 7: 26680-26691, 2016.

10. Washington $\mathrm{K}: 7$ th edition of the AJCC cancer staging manual: Stomach. Ann Surg Oncol 12: 3077-3079, 2010.

11. Livak KJ and Schmittgen TD: Analysis of relative gene expression data using real-time quantitative PCR and the 2(-Delta Delta C(T)) method. Methods 25: 402-408, 2001.

12. Lindsey BA, Markel JE and Kleinerman ES: Osteosarcoma overview. Rheumatol Ther 4: 25-43, 2017.

13. Isakoff MS, Bielack SS, Meltzer P and Gorlick R: Osteosarcoma: Current treatment and a collaborative pathway to success. J Clin Oncol 33: 3029-3035, 2015.

14. Wu J, Liao Q, He H, Zhong D and Yin K: TWIST interacts with $\beta$-catenin signaling on osteosarcoma cell survival against cisplatin. Mol Carcinog 53: 440-446, 2014.

15. He J, Xie Q, Xu H, Li J and Li Y: Circular RNAs and cancer. Cancer Lett 396: 138-144, 2017.

16. Qu S, Yang X, Li X, Wang J, Gao Y, Shang R, Sun W, Dou K and Li H: Circular RNA: A new star of noncoding RNAs. Cancer letters 365: 141-148, 2015.

17. Jin $H$, Jin $X$, Zhang $H$ and Wang W: Circular RNA hsa-circ-0016347 promotes proliferation, invasion and metastasis of osteosarcoma cells. Oncotarget 8: 25571-25581, 2017.

18. Li JF and Song YZ: Circular RNA GLI2 promotes osteosarcoma cell proliferation, migration, and invasion by targeting miR-125b-5p. Tumour Biol 39: 1010428317709991, 2017.

19. WITHDRAWN: Circular RNA hsa_circ_0001564 facilitates tumorigenesis of osteosarcoma via sponging miR-29c-3p. Tumour Biol 39: 1010428317709989, 2017.

20. Scholten DJ II, Timmer CM, Peacock JD, Pelle DW, Williams BO and Steensma MR: Down regulation of Wnt signaling mitigates hypoxia-induced chemoresistance in human osteosarcoma cells. PLoS One 9: e111431, 2014.

21. Li Z, Zhao L and Wang Q: Overexpression of long non-coding RNA HOTTIP increases chemoresistance of osteosarcoma cell by activating the Wnt/ $\beta$-catenin pathway. Am J Transl Res 8: 2385-2393, 2016.

22. He R, Liu P, Xie X, Zhou Y, Liao Q, Xiong W, Li X, Li G, Zeng Z and Tang H: circGFRA1 and GFRA1 act as ceRNAs in triple negative breast cancer by regulating miR-34a. J Exp Clin Cancer Res 36: 145, 2017.

23. Yang W, Du WW, Li X, Yee AJ and Yang BB: Foxo3 activity promoted by non-coding effects of circular RNA and Foxo3 pseudogene in the inhibition of tumor growth and angiogenesis. Oncogene 35: 3919-3931, 2016. 